\title{
Multiple sclerosis, human herpesvirus 4 and thyroid collision tumor: A case report
}

\author{
ANCA MARIA SIRBU ${ }^{1 *}$, CARMEN ADELLA SIRBU ${ }^{2,3}$, LUCIAN EFTIMIE $^{4-6 *}$, ANGELA MIRELA SOARE ${ }^{7}$, \\ MINERVA CLAUDIA GHINESCU $^{2 *}$ and FLORENTINA IONITA-RADU ${ }^{2,8}$ \\ ${ }^{1}$ Department of Endocrinology, 'C.I. Parhon’ National Institute of Endocrinology, 011863 Bucharest; \\ ${ }^{2}$ Department of Neurology, Faculty of Medicine, 'Titu Maiorescu' University, 031593 Bucharest; \\ ${ }^{3}$ Department of Neurology, 'Carol Davila' Central Military Emergency University Hospital, 010242 Bucharest; \\ ${ }^{4}$ Department of Pathology, 'Carol Davila' University of Medicine and Pharmacy, 050474 Bucharest; \\ ${ }^{5}$ Department of Pathology, 'Carol Davila' Central Military Emergency University Hospital, 010242 Bucharest; \\ ${ }^{6}$ Center for Microscopy-Microanalysis and Information Processing, Politehnica University of Bucharest, 77206 Bucharest; \\ ${ }^{7}$ Department of Neurology, 'Witting' Clinical Hospital, 010243 Bucharest; ${ }^{8}$ Department of Gastroenterology, \\ 'Carol Davila' Central Military Emergency University Hospital, 010242 Bucharest, Romania
}

Received May 29, 2020; Accepted June 29, 2020

DOI: $10.3892 /$ etm. 2020.8975

\begin{abstract}
The role of interferon $\beta-1 b$ (IFN $\beta-1 b)$, used for multiple sclerosis (MS) therapy, in cancer occurrence is uncertain. There is evidence supporting the role of human herpesvirus 4 [Epstein-Barr virus (EBV)] in thyroid cancer and MS. Simultaneous occurrence of papillary and medullary carcinomas is rare, and its association with MS in a young woman raises questions. A 46-year-old female patient was diagnosed with relapsing-remitting multiple sclerosis in 2008. In 2018, cervical MRI detected a thyroid nodule with right cervical adenopathy. Her thyroid function was normal, but increased calcitonin levels were found $(70.53 \mathrm{pg} / \mathrm{ml}$; normal value: $<9.82 \mathrm{pg} / \mathrm{ml}$ ). EBV serology tested positive. Paraclinical studies ruled out multiple endocrine neoplasia syndrome. Whole thyroid resection with whole cervical lymph node dissection was performed. To our knowledge, this is the first case that describes an association between MS and thyroid collision tumors. Histological examination ascertained both papillary and medullary thyroid cancer. After surgery, the calcitonin level normalized, and the patient received a therapeutic dose of iodine-131. IFN $\beta$-1b therapy was discontinued. The coexistence of thyroid cancers in MS patients could be explained by immune-mediated inflammation. Although EBV
\end{abstract}

Correspondence to: Professor Carmen Adella Sirbu, Department of Neurology, Faculty of Medicine, 'Titu Maiorescu' University, 67A Gheorghe Petraşcu Street, 031593 Bucharest, Romania

E-mail: sircar13@yahoo.com

${ }^{*}$ Contributed equally

Key words: thyroid collision tumors, papillary thyroid cancer, medullary thyroid carcinoma, multiple sclerosis, interferon $\beta-1 b$, disease-modifying therapy, thyroglobulin, immunohistochemistry, calcitonin, human herpesvirus 4 is not the only agent responsible for the development of MS or thyroid cancers, it could be considered a contributory factor in our case. Further research on EBV involvement in the occurrence of simultaneous immune pathologies in various organs is needed to confirm these data.

\section{Introduction}

Developing from the neural crest, both the brain and the thyroid can be affected by immunological imbalances. Possible associations of cancer with multiple sclerosis and immunomodulatory or immunosuppressant drugs have been investigated. The successive use of two or more disease-modifying therapies for MS patients can increase the risk of neoplasia (1). The results are contradictory regarding this topic, ranging between an increased risk of respiratory cancer, urinary system neoplasia, and nervous system cancer for MS patients and a lower risk of cancer in the MS population (2-5). One study found a higher incidence of thyroid cancer in MS cohort, but it did not specify the type of thyroid neoplasm implicated (1).

Recent studies have revealed that long-term immunosuppressive treatment for MS increases the risk of cancer (6,7). Thyroid neoplasia is the most frequent endocrine cancer, accounting for $\sim 2.1 \%$ of all malignancies (8). Differentiated thyroid cancer, including papillary and follicular types, evolving from thyroglobulin-secreting follicular cells, represents $\sim 80 \%$ of all thyroid neoplasia cases. Medullary carcinoma, which derives from calcitonin-producing parafollicular $\mathrm{C}$ cells, is relatively rare, accounting for 5-10\% (9). However, very rare combinations of two thyroid malignancies in the same patient have also been observed. Such cases are known as collision tumors, a rare entity described in the literature as the coexistence of at least two distinct tumors with different genetic origins and histologically distinct morphologies in the same organ and with no transition area between them. Such tumors have been described in the colon, lungs, ovaries, skin, and thyroid gland, with increasing 
incidence. Regarding the thyroid gland, the most frequent collision is that of papillary and medullary carcinomas (9). The role of the Epstein-Barr virus (EBV) is often discussed in relation to both MS and thyroid cancers, with a possible association between the two. Collision tumors must be considered more aggressive and posing a greater risk of recurrence compared with independent tumors. Therefore, monitoring cases is more complicated, as the evolution and risks of each tumor involved must be taken into account.

\section{Case report}

A 46-year-old female patient was admitted to our clinic in 2018. Her personal medical history included arterial hypertension with preeclampsia, with a first neurological episode of abdominal and crural paresthesia in 2003, when she was 31 years old, which spontaneously remitted within a month. Upon relapse in 2008 with the same symptoms, after a magnetic resonance imaging (MRI) examination, she was diagnosed with recurrent-remitting multiple sclerosis, with an Expanded Disability Status Scale (EDSS) score of 1 . Interferon $\beta-1 b$ (IFN $\beta-1 b)$ treatment was initiated at doses of $250 \mathrm{mg}$ (8.0 million IU) subcutaneously every other day. Her family medical history included breast cancer (maternal grandmother) and diabetes mellitus type 2 (father). The course of disease was good after 10 years of treatment, with no evidence of disease activity (NEDA 2): clinical activity, disability progression. In 2018, she was admitted for cervical pain, and cervical MRI examination showed a gadolinium enhancement of the right thyroid lobe. In October 2018, the patient was referred to the Endocrinology Unit for evaluation. No family history of thyroid cancer or multiple endocrine neoplasia (MEN) was reported. Her physical examination was normal. Moderately high serum calcitonin values $(70.53 \mathrm{pg} / \mathrm{ml}$; normal value: $<9.82 \mathrm{pg} / \mathrm{ml}$ ) were detected. The patient had normal thyroid function (thyroid-stimulating hormone [TSH)]: $1.65 \mu \mathrm{IU} / \mathrm{ml}$; normal range: $0.5-4.5 \mu \mathrm{IU} / \mathrm{ml}$ ) and no alteration of calcium metabolism. As her serum parathormone, fractionated plasma, and 24-h urinary total metanephrines were normal, MEN syndrome was excluded. Her serum $25(\mathrm{OH})$ vitamin D level was $14.7 \mathrm{ng} / \mathrm{ml}$ (normal value: $>20 \mathrm{ng} / \mathrm{ml}$ ). Her immunoglobulin $\mathrm{G}(\mathrm{IgG})$ antibodies against $\mathrm{EB}$ viral capsid antigen were positive $>750 \mathrm{U} / \mathrm{ml}$ (negative $<20 \mathrm{U} / \mathrm{ml}$ ), while her anti-EBV nuclear antigen antibody IgG value was $476 \mathrm{E} / \mathrm{ml}$ (normal value $<5$ ), indicating a prior EBV infection. RET and BRAF V600E gene mutations were not detected.

Ultrasound examination revealed two nodules in the right thyroid lobe: one in the middle third of the posterior part, hypoechoic $(14.5 \times 16.9 \times 8.7 \mathrm{~mm})$, with irregular margins, showing micro- and macrocalcifications, with chaotic vascularity in the entire nodule in color Doppler evaluation; the other inferior and lateral to it, a hypoechoic mass $(11 \times 10.2 \times 7.9 \mathrm{~mm})$ with regular margins and moderately perinodular flow signals.

The dominant nodule was classified as highly suspected for malignancy. Based on her serum calcitonin level, the patient was referred for surgery. Total thyroidectomy with central compartment cervical lymph node dissection was performed in November 2018. Histopathological examination of the resected specimen identified a collision tumor with a combination of papillary carcinoma and medullary microcarcinoma within the right lobe of the thyroid gland. Medullary thyroid microcarcinoma is a neuroendocrine tumor derived from $\mathrm{C}$ cells (formerly called parafollicular cells) of the ultimobranchial body of the neural crest, which secrete calcitonin, usually located at the junction of the upper and middle portions of the thyroid lobes. This tumor can mimic any other thyroid malignancy of microscopic description. Immunohistochemical examination showed that the patient's medullary thyroid microcarcinoma tumor cells stained positive weak-to-moderate for calcitonin and strong positive for generic neuroendocrine markers (i.e., chromogranin), thyroid transcription factor 1, and carcinoembryonic antigen (Fig. 1) $(10,11)$. One-year follow-up after thyroidectomy showed no evidence of recurrent disease (structural or biochemical).

The study was approved by the Ethics Committee of 'Carol Davila' Central Military Emergency University Hospital (Bucharest, Romania) and the patient's informed consent was obtained.

\section{Discussion}

Due to the suspected medullary carcinoma, and because of the preoperatively high level of calcitonin, whole thyroidectomy with central lymph node resection was performed (12). As a consequence of total thyroidectomy through throat dissection, hypoparathyroidism, either transitory or permanent (due to inadequate surgical techniques, local hematoma, blood flow disturbances, or direct glandular lesions) may occur. Some authors have recommended autotransplantation of at least two parathyroid glands in such cases $(13,14)$. After surgery, our patient developed laryngeal diplegia, with the left vocal cord immobilized in the paramedian position and the right vocal cord exhibiting a discrete adduction movement with sufficient respiratory space. The laryngeal nerve palsy recovered within a few months.

Neurological monitoring during thyroidectomy reveals useful information regarding the integrity and functionality of the recurrent laryngeal nerves. In this context, two-stage thyroidectomy is useful (15). Another surgical procedure to preserve the recurrent laryngeal nerve is robotic-assisted breast-axillo insufflation thyroidectomy (RABIT), which allows a simultaneous and symmetrical visualization and an easier approach (16). After surgery, our patient's serum calcitonin levels returned to normal levels ( $<2 \mathrm{pg} / \mathrm{ml}$; normal value: $<11.5 \mathrm{pg} / \mathrm{ml})$, which indicated that there was no residual tumor tissue. For the papillary cancer with microscopic invasion into the perithyroidal soft tissue, the patient was administered radioiodine (50 mCi of iodine-131). Thyroid hormone withdrawal was induced six weeks prior to radioablation. At that time, biochemical tests of the patient's thyroid status showed the following values: $\mathrm{TSH}>47.6 \mu \mathrm{IU} / \mathrm{ml}$ (normal range, $0.5-4.5$ ), stimulated thyroglobulin $0.5 \mathrm{ng} / \mathrm{ml}$, and anti-thyroglobulin antibodies $<1 \mathrm{IU} / \mathrm{ml}$ (normal range, $0-4$ ).

One year after surgery and radiotherapy, the patient was well, with hormonal levels within normal ranges and no evidence of local recurrence on ultrasound. No clinical or imaging signs of MS progression were detected, despite the discontinuation of immunomodulatory treatment with IFN $\beta-1 b$. Simultaneous occurrences of two or more cancers in the thyroid or in multiple organs have been described in immunosuppressed patients, some with autoimmune diseases, previously administered immunosuppressive treatments $(17,18)$. 

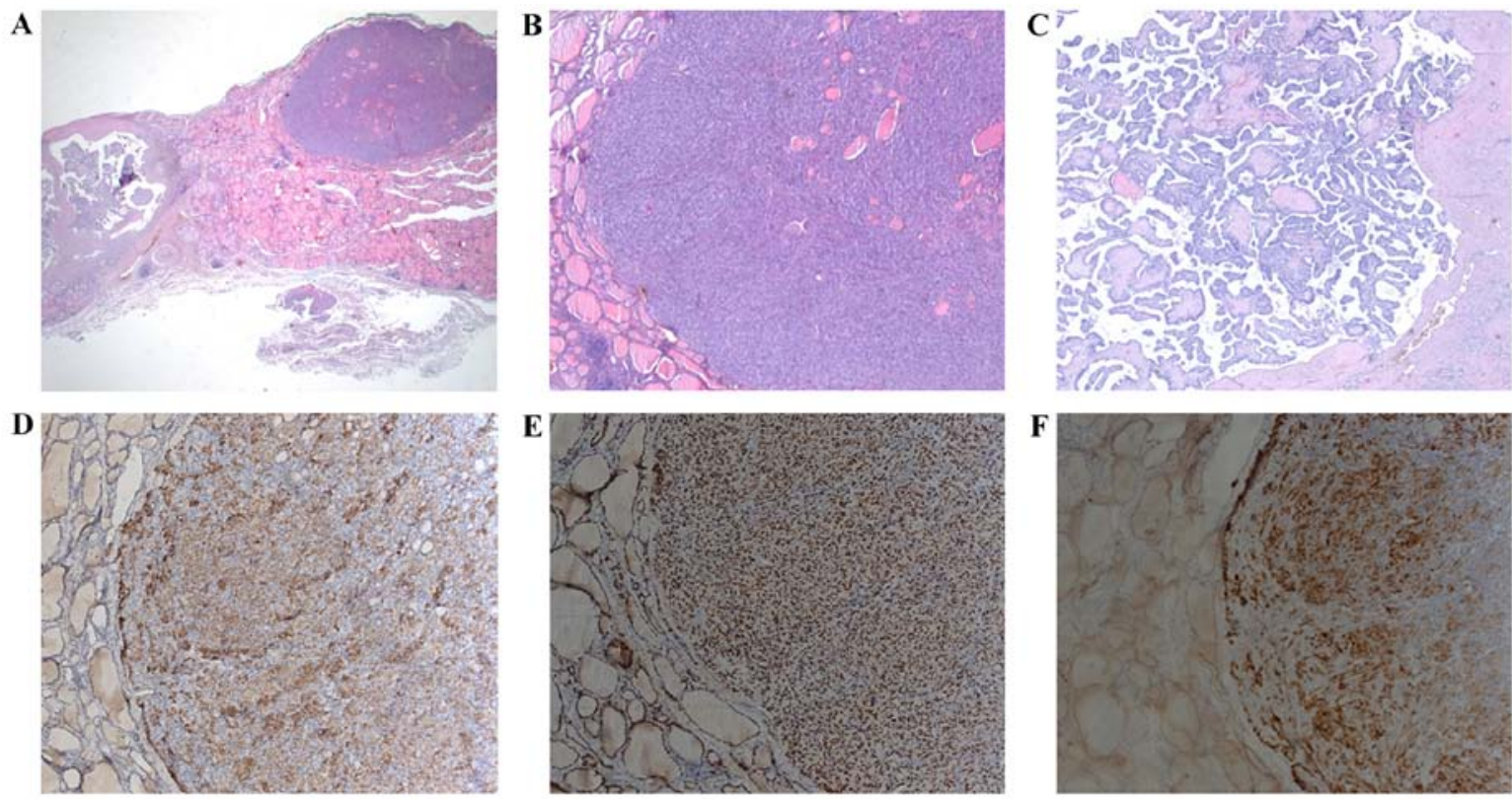

Figure 1. (A) Hematoxylin and eosin stain. Original magnification, x10. First nodule (9x5 mm): medullary thyroid microcarcinoma (right; pT1a N0 Mx). This type is represented in the International Classification of Diseases for Oncology (ICD-O) by the code 8345-3. Second marginal nodule (17 mm): papillary thyroid carcinoma (left; pT1b N0 Mx L1). This type is represented in the International Classification of Diseases for Oncology (ICD-O) by the code 8260-3. (B) Medullary thyroid microcarcinoma. Hematoxylin and eosin stain. Original magnification, $x 50$. This type of tumor, measuring $<1 \mathrm{~cm}$ in diameter, exhibits a nesting, solid, and trabecular growth pattern. The tumor cells are round, polygonal, and focal spindle-shaped, with round nuclei with coarsely clumped chromatin and eosinophilic-to-amphophilic granular cytoplasm due to secretory granules. (C) papillary thyroid carcinoma. Hematoxylin and eosin stain Original magnification, x50. This type of infiltrating, partially encapsulated tumor exhibits a papilliferous growth pattern, composed of randomly oriented papillae with a complex arborizing pattern, which are formed by a central fibrovascular stalk covered by a neoplastic epithelial lining, together with the presence of well-formed psammoma bodies or in an early stage of formation. These tumor cells have typical nuclear features and display a characteristic set of abnormalities divided into three major categories: nuclear enlargement and overlapping, circumferential irregularities of the nuclear membrane (garlands), and nuclear pseudoinclusions that represent deep cytoplasmic invaginations into the nucleus. Immunohistochemical staining shows that medullary thyroid microcarcinoma tumor cells stain consistently positive weak-to-moderate for calcitonin and strong positive for generic neuroendocrine markers (i.g., chromogranin), thyroid transcription factor 1 (TTF1), also called thyroid specific enhancer-binding protein, and carcinoembryonic antigen (CEA). (D) Chromogranin. Original magnification, x100. The patient's tumor cells showed strong positivity for chromogranin. (E) TTF1. Original magnification, x100. The patient's tumor cells showed strong positivity for TTF1. (F) CEA. Original magnification, x100. The patient's tumor cells showed strong positivity for CEA.

Exposure to IFN $\beta-1 b$ is not associated with an increased risk of neoplasia (19). It is estimated that infective agents are implicated in 2 million cancers yearly, $10 \%$ of which are due to EBV (20). EBV, also known as human herpesvirus 4 (HH4), is found in over $95 \%$ of the general population, transmitted through saliva. EBV infects the B lymphocytes, reducing gene expressions from $\sim 100$ to just 9 proteins, and has the ability to hide and remain in a latent state for years (21). While the incorporation of viral DNA in that of the host cell is well known, the oncogenesis mechanism has not yet been identified (22). EBV genome examination by polymerase chain reaction detected EBV DNA in $71.9 \%$ of a thyroid cancer patient cohort. Other authors reported similar findings $(23,24)$. Another study, however, did not confirm the association between thyroid tumors and the presence of EBV (25). EBV involvement in MS etiopathogenesis has been extensively studied (26). B lymphocytes hosting EBV are involved in triggering aberrant immune responses in multiple sclerosis, associated with genetic predisposition and environmental factors as a background. The EBV involvement in MS etiopathogenesis have been revealed by serological studies and the detection of the virus in patients' brains (27).

However, vitamin D deficiency, confirmed in our case also correlates with thyroid cancers and MS. Meta-analyses have associated an optimum level of $25(\mathrm{OH})$ vitamin D with a low risk of thyroid cancer $(28,29)$. Although vitamin D levels are associated with increased MS, the role of supplement doses should be further investigated (30). Difficulties in supporting differential diagnosis, or in the medical or surgical approach of patients with chronic neoplastic conditions are inherent (31-34), but early detection and intervention increase survival and quality of life.

In conclusion, the coexistence of thyroid cancers in MS patients could be explained by an immune-mediated inflammation involved in both pathologies. Although EBV is not the only agent responsible for the development of MS or thyroid cancers, it could be considered a contributory factor in our case. Simultaneous onset of medullary and papillary thyroid carcinoma is rare. Treatment and follow-up strategies should be individualized according to the aggressiveness of tumors. Further research on EBV involvement in the occurrence of simultaneous immune pathologies in various organs is needed to confirm these data.

\section{Acknowledgements}

Not applicable.

\section{Funding}

No funding was received. 


\section{Availability of data and materials}

The datasets used and/or analyzed during the current study are available from the corresponding author on reasonable request.

\section{Authors' contributions}

AMSi, CAS, LE, AMSo, MCG and FIR were involved in the conception of the study. AMSi, CAS and LE contributed equally to the acquisition of the data and the drafting of the manuscript. AMSo, MCG and FIR contributed equally to the critical revisions of the manuscript for important intellectual content. All authors read and approved the final manuscript.

\section{Ethics approval and consent to participate}

The study was approved by the Ethics Committee of 'Carol Davila' Central Military Emergency University Hospital (Bucharest, Romania).

\section{Patient consent for publication}

The patient's informed consent was obtained.

\section{Competing interests}

The authors declare that they have no competing interests.

\section{References}

1. D'Amico E, Chisari CG, Arena S, Zanghì A, Toscano S Lo Fermo S, Maimone D, Castaing M, Sciacca S, Zappia M, et al Cancer risk and multiple sclerosis: Evidence from a large italian cohort. Front Neurol 10: 337, 2019.

2. Etemadifar M, Jahanbani-Ardakani H, Ghaffari S, FereidanEsfahani M, Changaei H, Aghadoost N, Jahanbani Ardakani A and Moradkhani N: Cancer risk among patients with multiple sclerosis: A cohort study in Isfahan, Iran. Caspian J Intern Med 8: 172-177, 2017.

3. Gaindh D, Kavak KS, Teter B, Vaughn CB, Cookfair D, Hahn T and Weinstock-Guttman B; New York State Multiple Sclerosis Consortium: Decreased risk of cancer in multiple sclerosis patients and analysis of the effect of disease modifying therapies on cancer risk. J Neurol Sci 370: 13-17, 2016.

4. Grytten N, Myhr KM, Celius EG, Benjaminsen E, Kampman M, Midgard R, Vatne A, Aarseth JH, Riise T and Torkildsen Ø: Risk of cancer among multiple sclerosis patients, siblings, and population controls: A prospective cohort study. Mult Scler: Oct 1, 2019 (Epub ahead of print). doi: 10.1177/1352458519877244.

5. Moisset X, Perié M, Pereira B, Dumont E, Lebrun-Frenay C, Lesage FX, Dutheil F, Taithe F and Clavelou P: Decreased prevalence of cancer in patients with multiple sclerosis: A case-control study. PLoS One 12: e0188120, 2017.

6. Lebrun $\mathrm{C}$ and Rocher F: Cancer risk in patients with multiple sclerosis: Potential impact of disease-modifying drugs. CNS Drugs 32: 939-949, 2018

7. Ragonese P, Aridon P, Vazzoler G, Mazzola MA, Lo Re V, Lo Re M, Realmuto S, Alessi S, D'Amelio M, Savettieri G, et al: Association between multiple sclerosis, cancer risk, and immunosuppressant treatment: A cohort study. BMC Neurol 17: 155, 2017.

8. Grimm D: Current knowledge in thyroid cancer-from bench to bedside. Int J Mol Sci 18: 1529, 2017.

9. Thomas VP and George R: Collision tumors of the thyroid: Review of literature and report of a case of papillary-follicular collision tumor. Thyroid Res Pract 15: 60-64, 2018.

10. Lloyd RV, Osamura RY, Kloppel G and Rosai J: WHO Classification of Tumours of Endocrine Organs. 4th edition. IARC, Lyon, pp65-114, 2017.

11. Rosai J, BeLellis RA, Carcangiu ML, Frable WJ and Tallini G: Tumors of the thyroid and parathyroid glands. In: AFIP Atlas of Tumor Pathology. ARP, Maryland, pp103-130, 2014.
12. Park YM, Kim JR, Oh KH, Cho JG, Baek SK, Kwon SY, Jung KY and Woo JS: Comparison of functional outcomes after total thyroidectomy and completion thyroidectomy: Hypoparathyroidism and postoperative complications. Auris Nasus Larynx 46: 101-105, 2019.

13. Ritter K, Elfenbein D, Schneider DF, Chen H and Sippel RS: Hypoparathyroidism after total thyroidectomy: Incidence and resolution. J Surg Res 197: 348-353, 2015.

14. Teshima M, Otsuki N, Morita N, Furukawa T, Shinomiya H, Shinomiya $\mathrm{H}$ and Nibu KI: Postoperative hypoparathyroidism after total thyroidectomy for thyroid cancer. Auris Nasus Larynx 45: 1233-1238, 2018.

15. Christoforides C, Papandrikos I, Polyzois G, Roukounakis N, Dionigi $\mathrm{G}$ and Vamvakidis K: Two-stage thyroidectomy in the era of intraoperative neuromonitoring. Gland Surg 6: 453-463, 2017.

16. Nayak SP, Sadhoo A, Gangadhara B, Reddy S, Khan A, Munisiddaiah D and Ramakrishnan A: Robotic-assisted breastaxillo insufflation thyroidectomy (RABIT): A retrospective case series of thyroid carcinoma. Int J Clin Oncol 25: 439-445, 2020.

17. Milosevic Z, Tanic N, Bankovic J, Stankovic T, Buta M, Lavrnic D, Milovanovic Z, Pupic G, Stojkovic S, Milinkovic V, et al: Genetic alterations in quadruple malignancies of a patient with multiple sclerosis: Their role in malignancy development and response to therapy. Int J Clin Exp Pathol 7: 1826-1833, 2014.

18. Roshini AP, Ramesh R and Rajalakshmi T: HATRICKsynchronous triple primary tumors of thyroid. Indian J Surg Oncol 9: 592-594, 2018

19. Kingwell E, Evans C, Zhu F, Oger J, Hashimoto S and Tremlett H: Assessment of cancer risk with $\beta$-interferon treatment for multiple sclerosis. J Neurol Neurosurg Psychiatry 85: 1096-1102, 2014.

20. Young LS, Yap LF and Murray PG: Epstein-Barr virus: More than 50 years old and still providing surprises. Nat Rev Cancer 16: 789-802, 2016.

21. Fugl A and Andersen CL: Epstein-Barr virus and its association with disease - a review of relevance to general practice. BMC Fam Pract 20: 62, 2019.

22. Pyzik A, Grywalska E, Matyjaszek-Matuszek B, Ludian J, Kiszczak-Bochyńska E, Smoleń A, Roliński J and Pyzik D: Does the Epstein-Barr virus play a role in the pathogenesis of Graves' disease? Int J Mol Sci 20: 3145, 2019.

23. Bychkov A and Keelawat S: Epstein-Barr virus and thyroid cancer: The controversy remains. J Endocrinol Invest 40: 891-892, 2017.

24. Moghoofei M, Mostafaei S, Nesaei A, Etemadi A, Sadri Nahand J, Mirzaei H, Rashidi B, Babaei F and Khodabandehlou N: Epstein-Barr virus and thyroid cancer: The role of viral expressed proteins. J Cell Physiol 234: 3790-3799, 2019.

25. Yu ST, Ge JN, Li RC, Wei ZG, Sun BH, Jiang YM, Luo JY, Liu $\mathrm{H}$ and Lei ST: Is Epstein-Barr Virus infection associated with thyroid tumorigenesis? a southern China cohort study. Front Oncol 9: 312, 2019.

26. Guan Y, Jakimovski D, Ramanathan M, Weinstock-Guttman B and Zivadinov R: The role of Epstein-Barr virus in multiple sclerosis: From molecular pathophysiology to in vivo imaging. Neural Regen Res 14: 373-386, 2019.

27. Bar-Or A, Pender MP, Khanna R, Steinman L, Hartung HP, Maniar T, Croze E, Aftab BT, Giovannoni G and Joshi MA: Epstein-Barr virus in multiple sclerosis: Theory and emerging immunotherapies. Trends Mol Med 26: 296-310, 2020.

28. Hu MJ, Zhang Q, Liang L, Wang SY, Zheng XC, Zhou MM, Yang YW, Zhong Q and Huang F: Association between vitamin D deficiency and risk of thyroid cancer: A case-control study and a meta-analysis. J Endocrinol Invest 41: 1199-1210, 2018.

29. Kim D: The role of vitamin D in thyroid diseases. Int J Mol Sci 18: 1949, 2017.

30. Sintzel MB, Rametta M and Reder AT: Vitamin D and multiple sclerosis: A comprehensive review. Neurol Ther 7: 59-85, 2018.

31. Tomescu D, Cobilinschi C, Tincu RC, Totan A, Neagu TP, Diaconu CC, Tiglis M, Bratu OG and Macovei RA: Changes of thyroid hormonal status in organophosphate exposure. A systematic literature review. Rev Chim 69: 3364-3366, 2018.

32. Marcu DR, Ionita Radu F, Iorga LD, Manea M, Socea B, Scarneciu I, Isvoranu G, Costache R, Diaconu CC and Bratu OG: Vascular involvement in primary retroperitoneal tumors. Rev Chim 70: 445-448, 2019.

33. Marcu RD, Diaconu CC, Constantin T, Socea B, Ionita-Radu F, Mischianu DL and Bratu OG: Minimally invasive biopsy in retroperitoneal tumors. Exp Ther Med 18: 5016-5020, 2019. (Review).

34. Bratu OG, Diaconu CC, Mischianu DL, Constantin T, Stanescu AM, Bungau SG, Ionita-Radu F and Marcu RD: Therapeutic options in patients with biochemical recurrence after radical prostatectomy. Exp Ther Med 18: 5021-5025, 2019. 